\title{
Mössbauer Investigation of Spin Arrangements in $\mathrm{Er}_{2-x} \mathrm{Ce}_{x} \mathrm{Fe}_{14} \mathrm{~B}$
}

\author{
B.F. BogaCz* AND A.T. PȨDZIWIATR \\ M. Smoluchowski Institute of Physics, Jagiellonian University \\ Reymonta 4, 30-059 Kraków, Poland \\ It was theoretically postulated earlier that in compounds \\ $\mathrm{Er}_{2-x} \mathrm{Ce}_{x} \mathrm{Fe}_{14} \mathrm{~B}$ it may be possible to observe not only axial and pla- \\ nar spin arrangements but also a conical one. In order to experimentally \\ verify this hypothesis, ${ }^{57} \mathrm{Fe}$ Mössbauer spectroscopy analysis was used to \\ study the polycrystalline compounds $\mathrm{Er}_{2-x} \mathrm{Ce}_{x} \mathrm{Fe}_{14} \mathrm{~B}$ in the postulated \\ composition region $(x=1.0,1.1,1.2,1.3)$ in the wide range of temperatures. \\ The obtained experimental data do not clearly support the suggestion \\ of conical arrangement occurrence in the postulated compositions. They \\ indicate that such phenomenon may be shifted towards higher Ce content.
}

PACS numbers: $76.80 .+\mathrm{y}, 75.25 .+\mathrm{z}$

\section{Introduction}

The intermetallic compounds based on $\mathrm{Er}_{2} \mathrm{Fe}_{14} \mathrm{~B}$ have a tetragonal crystal lattice with the $P 4_{2} / m n m$ space group and belong to the $\mathrm{Nd}_{2} \mathrm{Fe}_{14} \mathrm{~B}$ structure type, in which there are two non-equivalent positions of $\mathrm{Nd}$ ions (4f and $4 \mathrm{~g}$ ), six positions of $\mathrm{Fe}$ ions $\left(16 \mathrm{k}_{1}, 16 \mathrm{k}_{2}, 8 \mathrm{j}_{1}, 8 \mathrm{j}_{2}, 4 \mathrm{c}, 4 \mathrm{e}\right)$ and one position of $\mathrm{B}$ atoms (4g) [1].

From the point of view of fundamental studies, one of the most interesting properties in this kind of compounds is the spin reorientation process occurring in the selected compositions: the direction of easy magnetization vector is changing from planar (in basal plane) to axial (along the $c$ axis) with increasing temperature. This is the result of a competition between axial and planar tendencies in Fe and Er sublattices [2]. This phenomenon was studied previously by different groups for different rare earth ions [3-8]. The study via single crystal neutron diffraction of $\mathrm{Er}_{2} \mathrm{Fe}_{14} \mathrm{~B}$ revealed that there is a change of crystal structure to orthorhombic below the spin reorientation transition [9].

Our previous investigations of reorientation process in $\mathrm{Er}_{2-x} \mathrm{Ce}_{x} \mathrm{Fe}_{14} \mathrm{~B}$ $(x=0.0,0.25,0.5,1.0)$ compounds $[10]$ yielded temperatures of reorientation, $T_{\mathrm{SR}}$,

*corresponding author; e-mail: bogdan.bogacz@if.uj.edu.pl 
in quite good agreement with the theoretical calculations based on the phenomenological model of Yamada et al. [11]. This model suggests that for $\mathrm{Er}_{2-x} \mathrm{Ce}_{x} \mathrm{Fe}_{14} \mathrm{~B}$ compounds it is possible to observe not only planar and axial spin arrangements but also a conical one for samples with greater contents of cerium. In order to experimentally verify this suggestion, several new compositions of $\mathrm{Er}_{2-x} \mathrm{Ce}_{x} \mathrm{Fe}_{14} \mathrm{~B}$ $(x=1.0,1.1,1.2,1.3)$ were prepared and studied.

The samples of $\mathrm{Er}_{2-x} \mathrm{Ce}_{x} \mathrm{Fe}_{14} \mathrm{~B}$ were prepared by means of induction melting stoichiometric proportions of the starting materials in high purity argon atmosphere and annealed at $900^{\circ} \mathrm{C}$ for two weeks. X-ray diffraction analysis performed at room temperature on random oriented powdered samples indicated the single phase character of materials.

The Mössbauer spectra of $\mathrm{Er}_{2-x} \mathrm{Ce}_{x} \mathrm{Fe}_{14} \mathrm{~B}$ were recorded in the temperature range $80-320 \mathrm{~K}$ (with a $5 \mathrm{~K}$ step in the vicinity of $T_{\mathrm{SR}}$ ) using a ${ }^{57} \mathrm{Co}(\mathrm{Rh})$ source and a computer driven constant acceleration mode spectrometer. The velocity scale was calibrated by a high purity iron foil. Isomer shifts were established with respect to the centre of gravity of the room temperature iron Mössbauer spectrum.

\section{Results and discussion}

A large number of ${ }^{57} \mathrm{Fe}$ Mössbauer spectra for $\mathrm{Er}_{2-x} \mathrm{Ce}_{x} \mathrm{Fe}_{14} \mathrm{~B}$ were measured in regions: below, during and above the transition. The Mössbauer spectra were analyzed with a transmission integral using exponential approximation of transmission integral [12] as a first step of evaluation. A procedure of simultaneous fitting of several spectra (up to ten) with interconnected parameters was applied, similarly as in our previous studies $[8,13]$. Such approach allows to assume a smooth temperature dependence of hyperfine interaction parameters; square for hyperfine magnetic field $-B$, linear for isomer shift - IS and quadrupole splitting - QS (QS was defined as $\left[\left(v_{6}-v_{5}\right)-\left(v_{2}-v_{1}\right)\right] / 2$, where $v_{i}$ are velocities corresponding to the Mössbauer line positions). One common set of three line widths was used for all Zeeman sextets. Exemplary spectra are presented in Fig. 1. The spectrum at the top of figure was recorded at the temperature below the spin reorientation, whereas the bottom one was obtained above the transition process. The three intermediate spectra represent spectra measured at temperatures inside the spin reorientation region. The spectra below and above the temperature region of spin reorientation were described using six Zeeman subspectra, called "low temperature" and "high temperature" Zeeman subspectra, respectively, with relative intensities according to the iron occupation of the crystallographic sublattices $\left(16 \mathrm{k}_{1}: 16 \mathrm{k}_{2}: 8 \mathrm{j}_{1}: 8 \mathrm{j}_{2}: 4 \mathrm{c}: 4 \mathrm{e}\right)$. Inside the temperature region of spin transition a coexistence of the "low" and the "high temperature" Zeeman subspectra was assumed, which, in consequence, gave twelve Zeeman sextets in the spectrum. This means that in the region of transition each subspectrum splits into two Zeeman sextets described by different hyperfine magnetic fields and different quadrupole splittings. In the course of transition, the "low" and "high temperature" Zeeman 


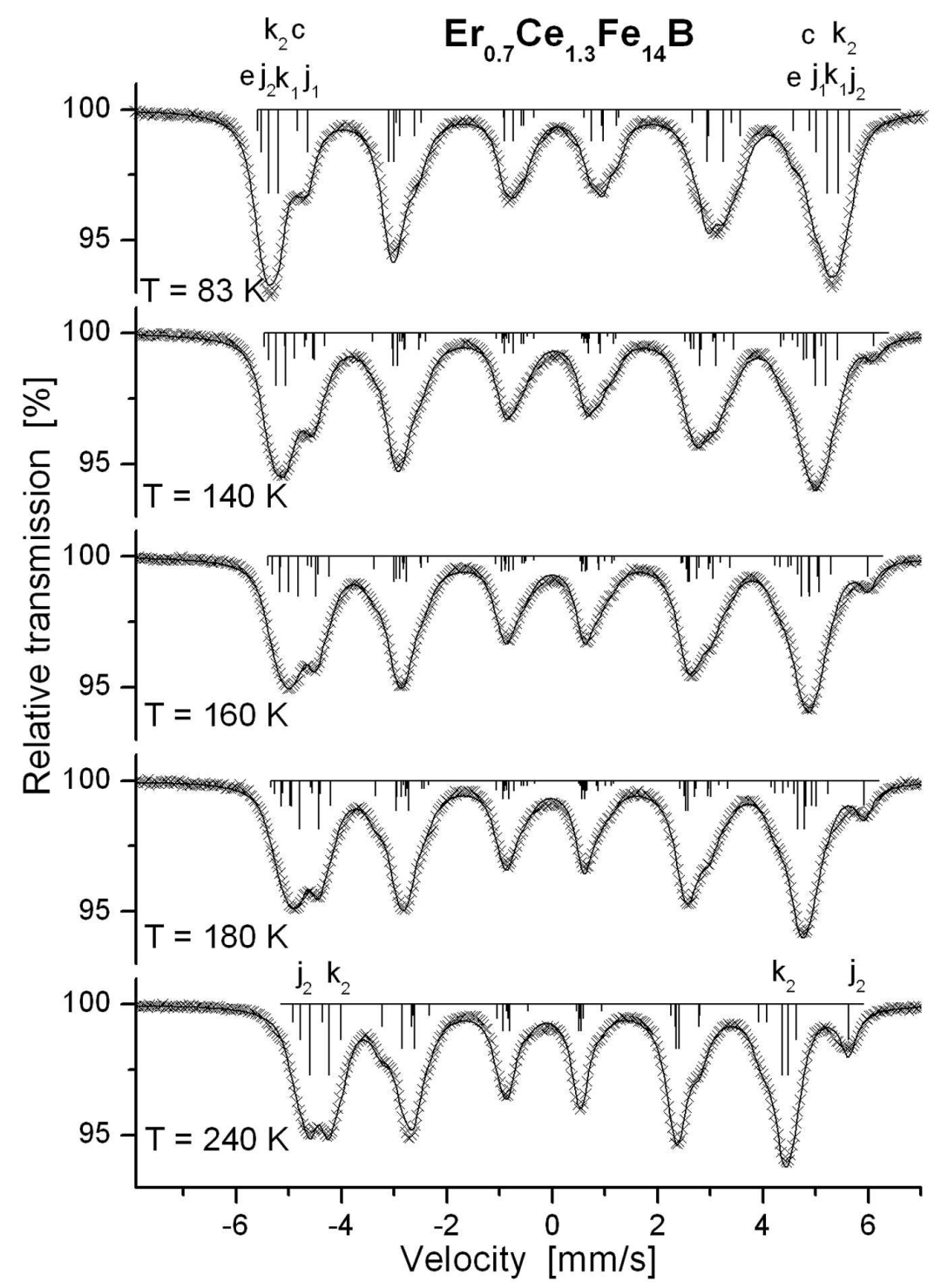

Fig. 1. Selected experimental ${ }^{57} \mathrm{Fe}$ Mössbauer spectra of $\operatorname{Er}_{2-x} \mathrm{Ce}_{x} \mathrm{Fe}_{14} \mathrm{~B}, x=1.3$ intermetallic compound. The solid lines are fits to the data. The stick diagrams show the line positions and their relative intensities.

sextets exchange gradually (between themselves) their contributions $C_{\mathrm{l}}$ and $C_{\mathrm{h}}$ to the total spectrum $\left(C_{\mathrm{l}}+C_{\mathrm{h}}=1\right)$. The clear separation of the sixth line of the sublattice $8 \mathrm{j}_{2}$ in the "high temperature" spectra (see Fig. 1) allows to follow this process in the simple way. The procedure of simultaneous fitting of several spectra permits the connection of hyperfine interaction parameters obtained for 


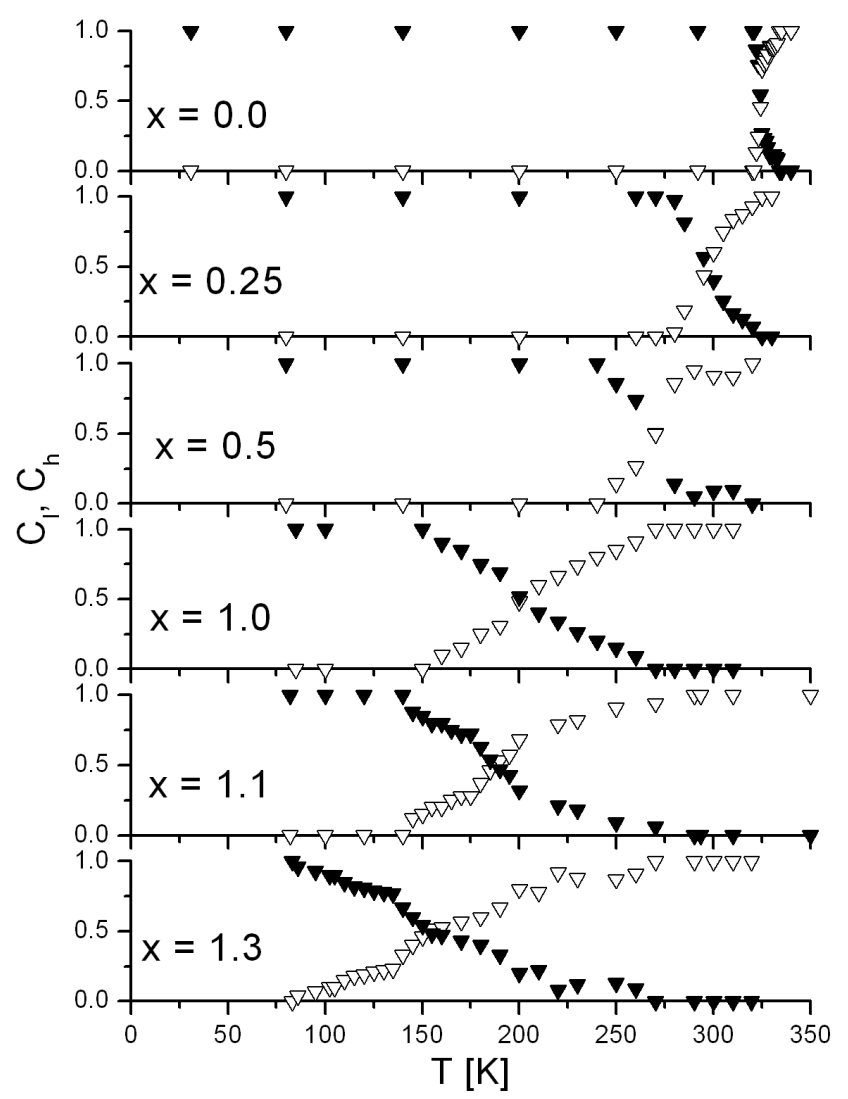

Fig. 2. The temperature dependences of subspectra contributions for $C_{1}$ - "low temperature" ( $\boldsymbol{\nabla})$ and $C_{\mathrm{h}}$ - "high temperature" $(\nabla)$ Zeeman sextets for $\operatorname{Er}_{2-x} \mathrm{Ce}_{x} \mathrm{Fe}_{14} \mathrm{~B}$.

the "low" and "high temperature" Zeeman sextets with the spectra parameters for the transition region. This makes easier to obtain an unambiguous description of experimental Mössbauer spectra and yields the contributions of the "low temperature" $-C_{\mathrm{l}}$ and the "high temperature" $-C_{\mathrm{h}}$ Zeeman subspectra to total spectrum. The spin reorientation temperatures from the Mössbauer studies, $T_{\mathrm{SR}}$, were taken as intersection points of $C_{\mathrm{l}}$ and $C_{\mathrm{h}}$ curves (Fig. 2).

A common linear temperature dependences of isomer shift IS caused by the second order Doppler shift effect were assumed for "low temperature" and "high temperature" Zeeman sextets (see example in Fig. 3). A square polynomial temperature dependences were taken into account for all hyperfine magnetic fields $B$. The possibility of a change in the value of $B$ during reorientation process was predicted. Figure 4 shows the example temperature dependences of the hyperfine field for the $8 \mathrm{j}_{2}$ sublattice. A small jump of $B$ value $\left(\Delta B \approx 1.1 \mathrm{~T}\right.$ for $8 \mathrm{j}_{2}$ sublattice) does not change with the increase in Ce content. For the quadrupole splitting QS the temperature dependence is very weak, but the values of QS change visibly 


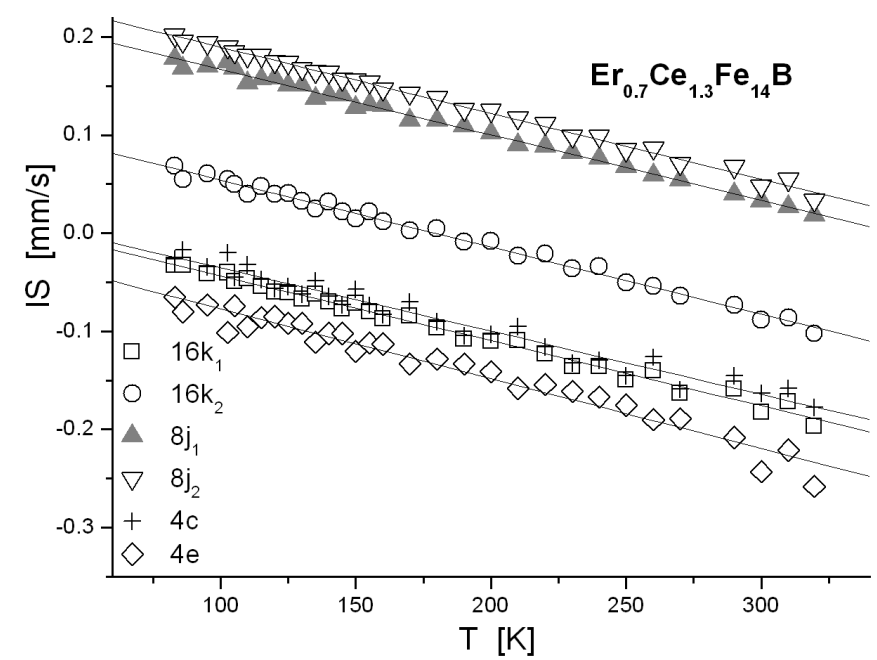

Fig. 3. The temperature dependence of isomer shift, IS, for different crystal sites of $\mathrm{Er}_{2-x} \mathrm{Ce}_{x} \mathrm{Fe}_{14} \mathrm{~B}, x=1.3$. The average error is $0.01 \mathrm{~mm} / \mathrm{s}$.

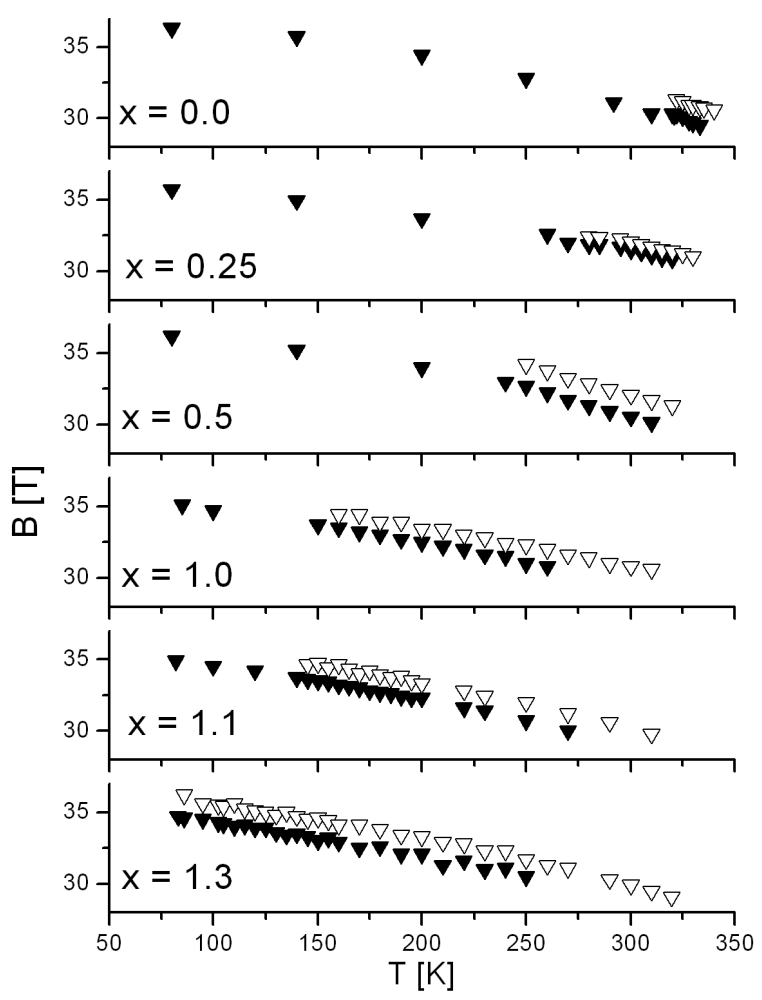

Fig. 4. The temperature dependences of the hyperfine field, $B$, for $8 \mathrm{j}_{2}$ crystal sites of $\mathrm{Er}_{2-x} \mathrm{Ce}_{x} \mathrm{Fe}_{14} \mathrm{~B}$. The average error is $0.1 \mathrm{~T}$. 


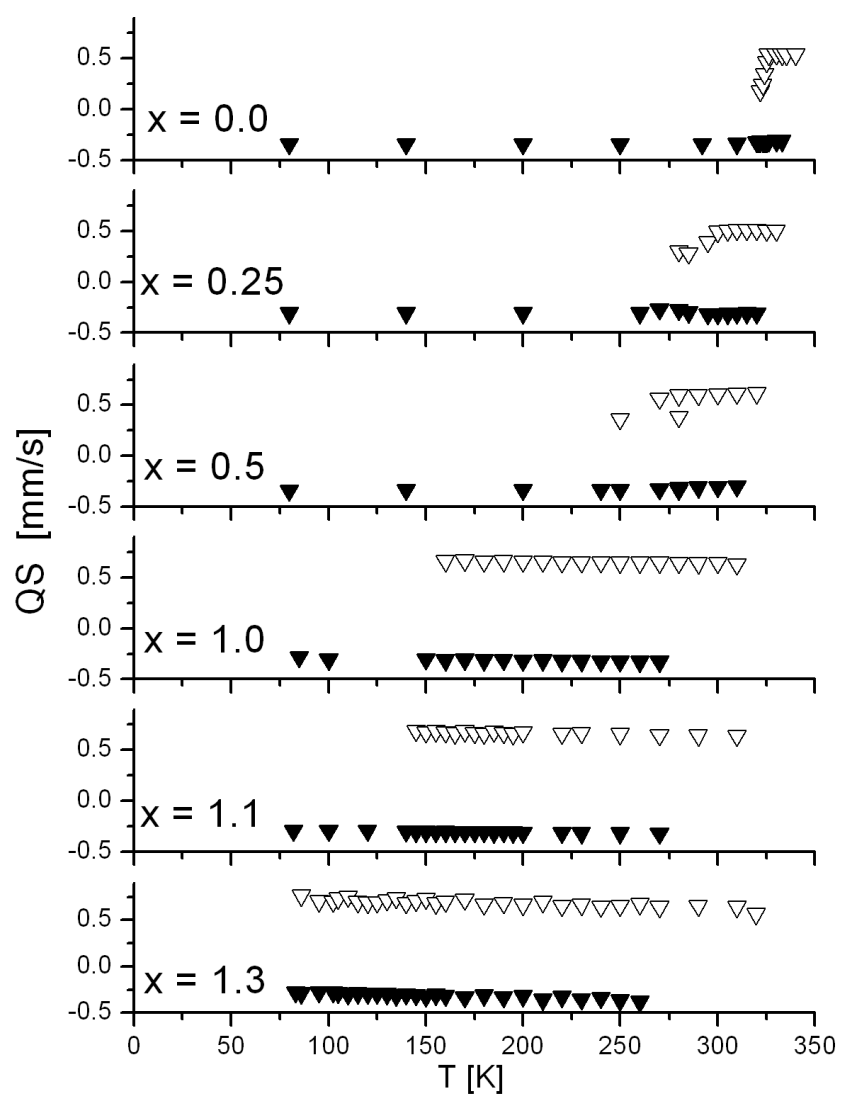

Fig. 5. The temperature dependences of the quadrupole splitting, QS, for $8 \mathrm{j}_{2}$ crystal sites of the $\operatorname{Er}_{2-x} \operatorname{Pr}_{x} \mathrm{Fe}_{14} \mathrm{~B}$. The average error is $0.01 \mathrm{~mm} / \mathrm{s}$.

in the reorientation process; they are different for "high" and "low temperature" sextets (see Fig. 5).

The behavior of QS is connected with the change of angle between the easy axis of magnetization and the electric field gradient (EFG) [14]. In the process of reorientation the magnetization vector changes its orientation from planar to axial direction $\left(90^{\circ}\right)$. The change of angle between the magnetization vector and the $V_{z z}$ axis of the EFG depends on the orientation of $V_{z z}$ with respect to the crystallographic axes which can be different for each sublattice [15]. Experimental results indicate that the values of QS jump from value for "low temperature" sextets to value for "high temperature" sextets without intermediate value QS for all sublattices Zeeman sextets in all new compounds $(x=1.0 \div 1.3)$. This suggests that in the investigated range of compositions the reorientation process occurs without conical arrangements of magnetic moments - on the contrary to theoretical prediction. This conclusion is supported by a good description of experimental Mössbauer spectra (see Fig. 1), visible also in the agreement between 
theoretical and experimental position of the sixth line of $8 \mathrm{j}_{2}$ Zeeman sextet in the whole temperature range of reorientation process. Therefore during the reorientation process with the increase in temperature a part of magnetic moments with axial orientation increases (see Fig. 2) but intermediate (conical) arrangements are omitted. Region of this process is represented by shaded area (coexistence of axial and planar arrangements) in the magnetic phase diagram (see Fig. 6). The ratio of QS values for $8 \mathrm{j}_{2}$ sublattice obtained below and above $T_{\mathrm{SR}}$ allows to conclude that the angle between $V_{z z}$ axis for this sublattice and the plane of reorientation of magnetic moment is smaller than $45^{\circ}$.

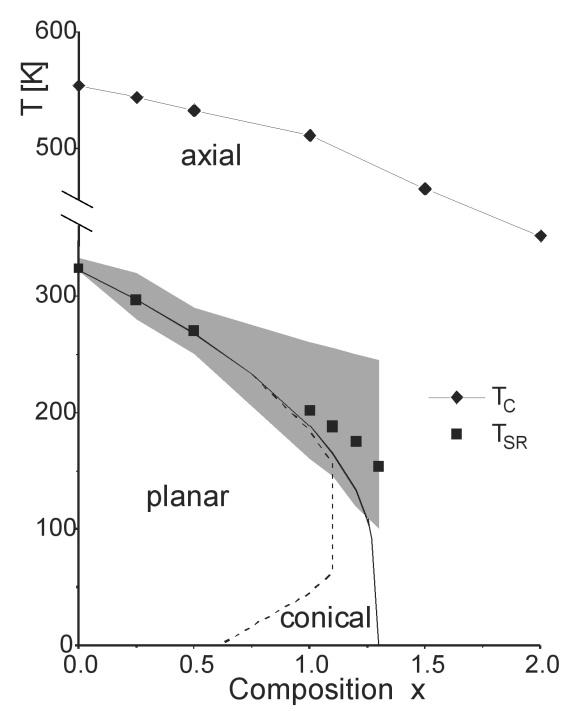

Fig. 6. Spin arrangement diagram for $\operatorname{Er}_{2-x} \mathrm{Ce}_{x} \mathrm{Fe}_{14} \mathrm{~B}$ system. $T_{\mathrm{c}}-$ Curie temperature, $T_{\mathrm{SR}}$ - spin reorientation temperature derived from the Mössbauer measurements. The solid and dashed lines represent the theoretically obtained limits of the range of the reorientation process. Between these lines the angle of spin orientation is $0^{\circ}<\theta<90^{\circ}$ (conical arrangement). The shaded area marks the experimentally estimated coexistence of axial and planar arrangements.

The reorientation temperatures $T_{\mathrm{SR}}$ determined from the new Mössbauer measurements are consistent with previous results (Fig. 6) but they are higher than the values predicted by the examined theoretical model. The width of temperature range of reorientation process systematically increases with the increase in $\mathrm{Ce}$ content in the compounds but any indication of conical arrangements of magnetic moments was not observed. It is possible that such phenomenon may be shifted towards higher Ce content. 


\section{References}

[1] J.F. Herbst, J.J. Croat, F.E. Pinkerton, W.B. Yelon, Phys. Rev. B 29, 4176 (1984).

[2] S. Hirosawa, Y. Matsuura, H. Yamamoto, S. Fujimura, M. Sagawa, H. Yamauchi, J. Appl. Phys. 59, 873 (1986).

[3] E. Burzo, Rep. Prog. Phys. 61, 1099 (1998).

[4] K.H.J. Buschow, in: Ferromagnetic Materials, Vol. 4, Eds. E.P. Wohlfarth, K.H.J. Buschow, Elsevier, Amsterdam 1988, p. 1.

[5] M.R. Ibarra, Z. Arnold, P.A. Algarabel, L. Morellon, J. Kamarad, J. Phys., Condens. Matter 4, 9721 (1992).

[6] A.T. Pȩdziwiatr, W.E. Wallace, J. Less-Common Met. 126, 41 (1996).

[7] C. Piqué, R. Burriel, J. Bartolomé, J. Magn. Magn. Mater. 154, 71 (1996).

[8] A.T. Pȩdziwiatr, B.F. Bogacz, A. Wojciechowska, S. Wróbel, J. Alloys Comp. 396, 54 (2005).

[9] P. Wolfers, M. Bacmann, D. Fruchart, J. Alloys Comp. 317-318, 39 (2001).

[10] A.T. Pȩdziwiatr, B.F. Bogacz, R. Gargula, Nukleonika 48, S59 (2003).

[11] M. Yamada, H. Kato, H. Yamamoto, Y. Nakagawa, Phys. Rev. B 38, 620 (1988).

[12] B.F. Bogacz, Mol. Phys. Rep. 30, 15 (2000).

[13] R. Wielgosz, A.T. Pȩdziwiatr, B.F. Bogacz, S. Wróbel, Mol. Phys. Rep. 30, 167 (2000).

[14] P. Gütlich, R. Link, A. Trautwein, Mössbauer Spectroscopy and Transition Metal Chemistry, Springer Verlag, Berlin 1978, p. 28.

[15] G.J. Long, F. Grandjean, Supermagnets - Hard Magnetic Materials, NATO ASI Series, Kluwer, Dordrecht 1990, p. 261. 